\title{
Kernos
}

Revue internationale et pluridisciplinaire de religion grecque antique

1 | 1988

Varia

\section{Vers une approche pluridisciplinaire de la religion grecque antique}

\section{Evanghelos A. Moutsopoulos}

\section{(2) OpenEdition \\ Journals}

\section{Édition électronique}

URL : http://journals.openedition.org/kernos/97

DOI : $10.4000 /$ kernos.97

ISSN : 2034-7871

\section{Éditeur}

Centre international d'étude de la religion grecque antique

\section{Édition imprimée}

Date de publication : 1 janvier 1988

ISSN : 0776-3824

\section{Référence électronique}

Evanghelos A. Moutsopoulos, "Vers une approche pluridisciplinaire de la religion grecque antique», Kernos [En ligne], 1 | 1988, mis en ligne le 31 janvier 2011, consulté le 06 mai 2019. URL : http:// journals.openedition.org/kernos/97 ; DOI : 10.4000/kernos.97 


\section{VERS UNE APPROCHE PLURIDISCIPLINAIRE DE LA RELIGION GRECQUE ANTIQUE. LE PROBLÈME DES SOURCES}

La connaissance des religions d'époques révolues repose, semble-t-il, sur quatre catégories de sources principales, qui en permettent des approches convergentes : (a) les textes sacrés; (b) les témoignages littéraires; (c) les témoignages artistiques; (d) les témoignages dits «parallèles», ces derniers se référant à des religions constituées ou impliquées qui remontent à des époques également révolues ou qui sont encore détectables de nos jours partout où la civilisation occidentale n'a pas, pour l'instant, réussi à s'infiltrer de manière catalytique pour faire disparaître la spécificité des sociétés traditionnelles. Chacune des catégories de sources énumérées présente, à son tour, plusieurs aspects dont l'analyse adéquate pourrait faire ressortir toute la richesse de renseignements relatifs à l'essence et aux modalités circonstancielles d'expression de chacune des religions en question. La rigueur méthodologique exige toutefois qu'au cours des approches respectives il soit tenu compte de l'unicité de la religion étudiée et que, malgré les classifications, les rapprochements et les typologies possibles, anachronismes, extrapolations et oppositions excessives soient exclus ou du moins évités.

(a) Les textes sacrés sont ceux qui nous sont parvenus avec le plus de parcimonie. Ils appartiennent à deux classes distinctes. Les uns, de par leur nature, se présentent sous la forme concise d'incantations, d'invocations, voire de formules carrément magiques, et dont la fonction consiste à provoquer l'action de la divinité à laquelle elles sont adressées. Quelques-uns des textes sacrés des Orphiques, par exemple, sont de cet ordre. Nous en connaissons de semblables, utilisés au cours de cérémonies de diverses sectes religieuses, en Grèce et ailleurs, notamment dans les religions d'aspect particulièrement mystique et orgiaque qui se sont développées en Thrace, en Asie mineure et au Proche Orient. Les autres ne sont, dans une large mesure, que des explicitations des premiers : leur fonction consiste à les expliquer et à les interpréter; d'où leur caractère exégétique, d'où aussi leur longueur. Ils sont manifeste- 
ment postérieurs aux précédents et visent à une certaine catéchèse d'adeptes tardifs dont il est utile de vaincre les réticences éventuelles. Plus que des oeuvres de caractère dogmatique, ils se présentent comme des récits, souvent comme des épopées qui racontent les hauts faits et les passions de la divinité, mais ne semblent servir qu'à une lecture édifiante et «à tête reposée», en tout cas comme préparation à quelque participation à des drômena dont les textes de la classe précédente sont les témoignages uniques. Des formes dramatiques, tel le dithyrambe, par exemple, constituent vraisemblablement des réalisations de type intermédiaire, voire transitoire : à la fois concis et étendus, selon le critère d'évaluation que l'on adopte, ils font partie de la phase préparatoire du culte avant d'y être intégrés, puis remplacés par des récits plus élaborés.

L'aspect le plus important qui est commun à toutes les formules cultuelles précises, c'est leur musicalité inhérente. Concises, pour être facilement mémorisées, ces formules sont musicales, notamment rythmiques, pour être scandées par l'assistance tout entière qui les répète sur invitation de l'officiant. Certaines d'entre elles se sont perpétuées une fois introduites dans l'art profane, et ont été adoptées par la tradition artistique, telle la formule $\hat{o}$ ton Adônin, qui a été intégrée en tant que clausule dans la strophe sapphique. Platon ne répète-t-il pas à cinq reprises, dans le seul livre II des Lois, que les chants (ôdai) sont, au fond, des incantations (épôdai)? Et, par ailleurs, carmen ne désignerait-il pas en latin le chant autant que le charme ? A n'en pas douter, il fut un temps où chant et incantation étaient confondus, rythme et mélodie étant, par ailleurs, confiés respectivement à des musiciens qui, sur le tard, devinrent des professionnels. Sur ce point, l'évolution du culte chrétien procure un témoignage éloquent. Le recours à l'art musical fut, de tous temps, une pratique hautement judicieuse, la musique exerçant un charme indéniable, propice à l'élévation du croyant et à son identification avec la divinité célébrée. Dans le dernier paragraphe de son Criton, Platon fait allusion aux cultes religieux de son époque; à l'en croire, l'adepte reconnaît, entre tous les airs, celui quii correspond à la divinité à laquelle il s'est voué. Et Aristide Quintilien, contemporain des Pères du IIe siècle, de soutenir, en interprétant la théorie platonicienne de la participation musicale, exposée dans le Timée, que l'âme humaine répond aux mouvements sonores par ses propres commotions, c'est-à-dire par des é-motions (mouvements) adéquates au genre de la musique qu'elle perçoit. 
(b) Les témoignages littéraires, nous venons d'en mentionner quatre exemples très typiques, mais qui sont bien loin de couvrir toute la gamme des possibilités qui s'offrent au chercheur. Ces témoignages vont de la simple mention ou allusion, à la description la plus détaillée - et encore, dans ce dernier cas, est-il nécessaire de savoir distinguer entre description authentique et mystification imaginaire, en passant par tous les degrés du processus de falsification. D'ailleurs, même dans le cas des allusions, on doit se montrer extrêmement circonspect, car on se trouve fréquemment face à des auteurs mal informés, sinon mal intentionnés, qui font état de pratiques propres à une secte donnée et qu'ils attribuent à une secte différente ou, pire, qui en arrivent à confondre les noms mêmes de sectes ou de pratiques totalement distinctes les unes des autres. A cet égard, il est donc utile de soumettre chaque témoignage à une critique minutieuse, avant d'en adopter le contenu. Les témoignages littéraires peuvent porter sur les faits suivants : dénomination de sectes; dénomination de divinités titulaires de cultes; structure des sectes et des cultes; pratiques cultuelles; textes sacrés, formules incantatoires, formes dramatiques, récits; lieux de culte, temples, processions, cérémonial, interdits (on sait, par exemple, que les membres de certaines familles dont l'origine était mal définie, donc contestée, étaient exclus de toute participation à la fête des Panathénées, et que cet interdit fut même appliqué à certains membres de la famille des Pisistratides).

On pourrait ajouter à ces catégories d'informations celles qui sont relatives aux croyances mêmes des adeptes. On se trouve, dès lors, placé sur un terrain extrêmement mouvant du fait qu'il n'existe aucunement, du moins dans le monde grec, de théologie officielle, donc de dogme, d'orthodoxie et, par voie de conséquence, d'hérésie. En effet, la notion d'hérésie, au sens actuel du terme, fut introduite avec le christianisme, en même temps que la notion de dogme théologique. On est ainsi en mesure de comprendre la richesse de la littérature religieuse grecque, notamment au niveau de la mythologie. Après les synthèses sur l'héortologie athénienne par A. Mommsen, et sur celle du reste de la Grèce par M. Nilsson, nous possédons un répertoire quasi complet des aspects officiels de la religiosité dans le monde.grec. Or, à côté de ces manifestations officielles répertoriées, il existe des allusions à des fêtes d'ordre orgiaque sur lesquelles il est d'usage que les auteurs anciens soient extrêmement taciturnes : ils couvrent les ténèbres de leur nature par 
les ténèbres des informations qu'il en offrent. Là encore, le moindre indice peut se révéler d'importance capitale. Certes, ainsi qu'il vient d'être admis, la religion grecque ne présente aucun côté dogmatique, théologique, au sens chrétien du terme; toutefois, l'adoption officielle de certains de ces aspects, comme le silence presque complet au sujet de certains autres, sont éloquents quant à l'existence d'une idéologie religieuse dominante. Partout où l'on a affaire à des cultes occultes, il est possible d'enrichir et de compléter nos connaissances en recourant aux indications indirectes que procurent aisément des pratiques parallèles qui, elles, sont mieux connues, mais à condition d'adopter également, en l'occurrence, une méthode critique permettant de prévenir confusions et interprétations erronées.

(c) Les témoignages artistiques. Cette classe de témoignages présente des aspects particulièrement variés. Outre les oeuvres d'art proprement dites, elle s'étend à des textes épigraphiques, tel le texte de l'hymne à Apollon découvert à Delphes et dont le déchiffrement n'a jusqu'ici donné aucune interprétation satisfaisante, au point qu'il est plus aisé, par exemple, de se faire une idée approximative de la musique religieuse de l'antiquité classique en recourant à des textes musicaux dont l'éthos remonte à l'époque hellénistique et dont la tradition orale byzantine a sauvegardé le style. Il manque encore la clef de déchiffrement définitif de ces textes, mais leur traitement selon les procédés actuels les plus avancés pourrait, sans nul doute, conduire prochainement à des résultats surprenants. A part ces documents tout à fait spéciaux, les témoignages artistiques susceptibles de faire avancer nos connaissances au sujet de la religion (ou des religions) de la Grèce antique peuvent être groupés dans l'une des deux catégories suivantes :

$\left.1^{\circ}\right)$ Lieux de culte naturels, aménagés ou construits : structures recouvrant de l'intérieur ou de l'extérieur une cavité ou autre accident de la nature associé à des pratiques religieuses, telles certaines constructions delphiques; temples demeurés presque intacts ou complètement détruits et ensevelis au cours des âges et que l'archéologue est appelé à remettre en lumière et à reconstituer; ensembles construits à l'intérieur d'une enceinte sacrée, tel le sanctuaire de Brauron qui, fouillé dans une large mesure, a permis de préciser le caractère totémique du culte local d'Artémis. 
$2^{\circ}$ ) Objets de culte, qui sont de nature très variée, et qui vont des simples ustensiles les plus rudimentaires et les plus ordinaires aux représentations les plus élaborées des symboles religieux intégrés dans chaque culte et, enfin, aux idoles et aux statues des diverses divinités, qui sont de véritables oeuvres d'art méritant d'être traitées comme telles. A côté de ces chefs-d'oeuvre, on appréciera des objets artistiques dont la décoration principale ou complémentaire fait allusion à telle institution ou à tel culte : certaines représentations de la frise du Parthénon se réfèrent directement à des aspects fondamentalement religieux de la fête des Panathénées; plusieurs stèles funéraires du Céramique font allusion à des cultes religieux peu connus par ailleurs : un seul détail suffit souvent à en rendre le message profondément révélateur, si bien qu'il se révèle lui-même plus qu'un détail, à savoir le centre de gravité noématique et sémantique, sinon artistique, du relief. Toutes ces indications concourent à clarifier le sens de tel ou tel culte ou de tel côté occulte de la religion grecque.

(d) Les témoignages «parallèles». La philosophie des religions et leur histoire comparée ont mis en évidence certaines affinités existant entre les religions apparemment les plus diverses. On n'oubliera pas que les pratiques religieuses des Grecs ont surgi à la suite d'un brassage étendu des éléments religieux indo-européens qui formaient le fond de leur culture d'origine avec ceux qu'ils ont empruntés aux sociétés autochtones préhelléniques ou aux peuples environnants d'origine également indoeuropéenne (Thraces en Europe; Phrygiens en Asie) ou sémitique (Proche Orient), et bien entendu, aux Égyptiens. Malgré le raffinement résultant de son intégration dans une culture et dans une civilisation elles-mêmes raffinées, la religion grecque présente un fond archaïque très proche $\mathrm{du}$ fond des ensembles de pratiques magico-religieuses propres aux sociétés archaiqques actuelles. Tout l'éventail des manifestations de l'irrationnel chez les Grecs, tel qu'il a été envisagé et répertorié par E. R. Dodds, témoigne de cette affinité. C'est en rapprochant la mentalité religieuse grecque de la mentalité des sociétés archaïques, telles qu'elle a été interprétée naguère par L. Lévy-Bruhl et, de nos jours, par $\mathrm{Cl}$. Lévi-Strauss et son école, par exemple, qu'on la pourra mieux comprendre. Quand on aura retrouvé le fond magique de la religion grecque, on en aura peut-être atteint l'aspect le plus important et, pour ainsi dire, le noyau. Toutes les autres couches n'en seraient que des développements ou des acquisitions ultérieurs qu'une analyse phénoménologique à rebours permettrait d'écarter 
successivement, pour arriver au même résultat par une voie différente.

Ce qui précède rend parfaitement clair le fait que pour mieux étudier la religion de la Grèce antique, il est nécessaire d'encourager la collaboration déjà existante, mais de manière encore lâche, entre philologues, archéologues et historiens de l'art, d'une part, et, d'autre part, entre historiens des religions, ethnologues, ethnomusicologues, sociologues, historiens de la philosophie et philosophes. Par la force des choses, la discipline pluridisciplinaire nouvelle ainsi établie ne saurait subsister au-delà d'une conception $a$ priori, ni, par conséquent, être effectivement viable et fiable sans être soutenue de fait par la convergence et la concordance des disciplines impérieusement invitées à collaborer au niveau de la recherche et de l'appréciation des objets communs fixés et des objectifs à atteindre. L'institution internationale qui tient ici même ses assises est appelée à devenir bientôt l'expression officielle principale, sinon unique, d'une telle collaboration.

Rue Hypsilantou 40

Evanghélos A. MOUTSOPOULOS

GR - 11521 ATHÈNES 